\title{
Evaluation of the Effectiveness of Cardiotocography vs USG Doppler in the Optimal Management of Intra Uterine Growth Restricted Fetuses
}

\author{
S. S. Gayathri ${ }^{1}$, T. H. Usha ${ }^{2}$ \\ ${ }^{1,2}$ Assistant Professor, Department of Obstetrics and Gynecology, Madurai medical college, Madurai
}

\begin{abstract}
Introduction: Intra uterine growth restricted fetuses need early detection of the hypoxic changes and timely intervention will have a great change in the improvement of the neonatal outcome. Material and Methods: A prospective type of comparative study was conducted in 100 antenatal mothers with singleton intra uterine growth restricted fetuses presenting more than 30 weeks gestation GROUP 1 monitored with Doppler and cardiotocography and GROUP 2 monitored with cardiotocography alone. To the group of IUGR fetuses and their detection rate with the neonatal outcome, compared. Results: In Doppler and CTG group, $70 \%$ of the cases were terminated in 34- 36 gestational age. In CTG alone group, 52 \% were terminated in 34-36 gestational age. Regarding Liquor status, oligohydramnios was associated in $56 \%$ of Doppler and CTG group and $66 \%$ in CTG alone group. $94 \%$ in Doppler CTG group and 72 $\%$ of the CTG alone group underwent cesarean section, among which $58 \%$ in both the groups had birth weight $<2 \mathrm{~kg}$ with, in Doppler and CTG group, $82 \%$ had APGAR > 7 and in CTG alone group, $90 \%$ had APGAR > 7. Conclusion: The Doppler velocimetry when compared with Cardiotocography is more advantageous in the surveillance of intra uterine growth restricted fetuses and in early decision making thereby in reduction of adverse neonatal outcomes.
\end{abstract}

Keywords: Doppler, cardiotocography, IUGR, surveillance, intervention

\section{Introduction}

The assessment of the growth of the fetus, health and their development are being thought to be the standard antenatal care. IUGR is defined as babies whose birth weight is being below the tenth percentile of the average for the corresponding gestational age. Incidence of IUGR in the term gestation babies is $5 \%$ where as Incidence of IUGR in the preterm gestation babies is more higher - 15\%.The IUGR babies are at greater risk for neonatal morbidity and mortality.The neonatal mortality rate of IUGR babies which are born at 38 weeks of gestation was $1 \%$ and its $0.2 \%$ in the babies with normal birth weight. This higher rate of neonatal morbidity and mortality necessitates earlier detection so that antepartum fetal surveillance methods can be applied appropriately to the infants and helpful for the babies to get proper institutional care at all levels and the neonatal .outcome can be improved. The methods of surveillance should be simple, safe, cheap, readily available and easily applicable to the study group. It must identify the right time for the appropriate management to reduce the morbidity and mortality.

\section{Aim}

The aim of the study is to find the risk factors, thereby to improve the outcome by timely intervention. It is the optimal decision forsurveillance and intervention that decides the outcome. andthe appropriate management reduces the morbidity and mortality.In this study, the CTG and the ultrasound Doppler methods of antepartum surveillance are been applied to the group of IUGR fetuses and compared their predictability in risk identification and improving the neonatal outcome.

\section{Materials and Method}

A Cross sectional - prospective study was conducted for a period of 1 year ( Jan2015 to Dec2015 ) at Government Rajaji Hospital, Madurai - Department of Obstetrics and Gynecology with collaboration of Department Of Radiology $\&$ Pediatrics on 100 antenatal mothers who presented to the department with singleton intra uterine growth restricted fetus, more than 30 weeks of gestation, excluding fetal malformation. The antenatal women were randomized into 2groups. GROUP 1 monitored with Doppler and cardiotocography and GROUP 2 monitored with cardiotocography alone.

In group 1, Doppler ultrasound done at time of entry into study and then proceeded weekly for the first 3 weeks. If normal, then done every 2 weeks and if abnormal, then continued weekly.Fetus with abnormal Doppler findings are terminated according to the severity and gestational age. Cardiotocography on alternate days for 20 minutes duration.. If found persistently non reactive, then pregnancy is terminated.

GROUP 2 monitored with Cardiotocography alone. Done on alternate days for 20 minutes duration. If found persistentlynon reactive, then pregnancy is terminated.

The outcomes measured are: The mode of delivery, the baby details namely sex of the baby, birth weight, one minute and five minute APGAR score, whether NICU care needed or not, any occurrence of neonatal complications and length of the NICU stay 
International Journal of Science and Research (IJSR)

ISSN (Online): 2319-7064

Index Copernicus Value (2015): 78.96 | Impact Factor (2015): 6.391

\section{Results}

In Doppler and CTG group, $74 \%$ of the antenatal mothers fall in 21-30 age group. In ctg alone group, $76 \%$ of the antenatal mothers fall in the $21-30$ age group.

Regarding Occupation, In Doppler and CTG group, $26 \%$ are in working group and rest are home makers. In CTG alone group, $28 \%$ fall in working group and the rest are home makers.

Considering obstetric code, In Doppler and CTG group, 56 $\%$ are primigravida and in CTG alone group, $70 \%$ are primigravida and rest are multigravida.

In Doppler and CTG group, $70 \%$ of the cases were terminated in 34- 36 gestational age. In CTG alone group, 52 $\%$ were terminated in 34-36 gestational age.

$52 \%$ of the antenatal mothers in Doppler and CTG group and $44 \%$ in CTG alone group were having gestational hypertension as risk factor associated with IUGR.

Regarding Liquor status, in Doppler and CTG group, oligohydramnios was associated in $56 \%$ of Doppler and CTG group and $66 \%$ in CTG alone group.

$94 \%$ in Doppler CTG group and $72 \%$ of the CTG alone group underwent cesarean section ( as in fig 1), among which $58 \%$ in both the groups had birth weight $<2 \mathrm{~kg}$ with, in Doppler and CTG group, $82 \%$ had APGAR > 7 and in CTG alone group, $90 \%$ had APGAR > 7

\begin{tabular}{|c|c|c|}
\hline Delivery mode & Doppler +CTG & CTG \\
\hline Labour Natural & 3 & 14 \\
\hline Caesarean & 47 & 36 \\
\hline
\end{tabular}

Figure 1

Regarding NICU admission, in Doppler CTG group, $96 \%$ got admitted in NICU and In CTG alone group, $80 \%$ got NICU admitted.

In Doppler and CTG group, the hypoxic changes were picked up in 36 among 50 and in CTG alone group, it was only 12 out of 50 were non reactive.( as in fig 2 )

\begin{tabular}{|c|c|c|}
\hline & Doppler + CTG & CTG \\
\hline Abnormal & 36 & 12 \\
\hline Normal & 14 & 38 \\
\hline
\end{tabular}

Figure 2

In Doppler and CTG group, among the 36 detected, 28 had NICU stay below 7 days and 8 had stay above 7 days (as in fig 3). In CTG alone group, among the 12 non reactive fetuses, 9 had NICU stay above 7 days and and 3 below 7 days (as in fig 4). This reveals that the detection by CTG at later stage is associated with poor neonatal outcome.

Doppler and CTG group Fig 3

\begin{tabular}{|c|c|c|}
\hline NICU stay & Abnormal (36) & Normal (14) \\
\hline$\leq 7$ days & 28 & 13 \\
\hline$>7$ days & 8 & 1 \\
\hline
\end{tabular}

\section{CTG Group Alone Fig4}

\begin{tabular}{|c|c|c|}
\hline NICU stay & Normal (38) & Abnormal(12) \\
\hline$\leq 7$ days & 33 & 3 \\
\hline$>7$ days & 5 & 9 \\
\hline
\end{tabular}

\section{Discussion}

Intra uterine growth restricted fetuses are more associated with the neonatal morbidity and mortality. It is the early detection of the hypoxic changes and timely intervention, that will have a dramatic change in the neonatal outcome. There are various methods of surveillance tools available to monitor intra uterine growth restricted fetuses. In this study, the Doppler velocimetry and Cardiotocography are been compared regarding their efficacy in the surveillance and optimal management of IUGR fetuses and their predictability of adverse neonatal outcome. The outcome parameters considered in this comparative study are cesarean section rate, birth weight, APGAR scores and length of NICU stay of the IUGR babies.

The study population were divided into 2 groups, with 50 antenatal mothers in each group.. In first group, IUGR was monitored by both Doppler and Cardiotocography. In second group, by only Cardiotocography. If any abnormality detected, the IUGR mothers have been managed as per protocol. Mode of Delivery and the baby details were recorded. Outcome of both the mother and baby were observed till they are discharged.

Age is an independant risk factor. In this study, the majority of the study population falls in $21-30$ yrs age and the mean is $25 \mathrm{yrs}$ on both the group. This is comparable with the mean age of $26.8 \mathrm{yrs}$ in study by DHARMAVIJAYA ET AL . The working group are very less in this study. Only $26 \%$ in Doppler and CTG group and $28 \%$ in CTG alone group belongs to the working group.

IUGR fetuses need higher institutional level care and better surveillance protocol. In this study, had $68 \%$ of the Doppler and CTG group and $56 \%$ of the CTG alone group were referral cases from various primary level health centers referred forhigher level neonatal care.

Among the study population, $56 \%$ of the Doppler and CTG group and $44 \%$ of the CTG alone group belong to primigravida category. In a similar study by Dharmavijaya ET AL it was $38.5 \%$ were primigravida. There are various causes and risk factors associated with intra uterine growth restriction, among which, in this study, the majority was gestational hypertension. $52 \%$ of the Doppler and CTG group and $44 \%$ of the CTG group had gestational hypertension associated. Gestational hypertension is a major risk factor associated with IUGR and the main pathophysiology leading to IUGR is Uteroplacental Insufficiency. All cases of gestational hypertension should be suspected for intra uterine growth restriction and be monitored cautiously. The patients with gestational hypertension should be under close surveillance so that IUGR can be detected earlier. Adequate BP control can have an added advantage in improvingmanagement.of IUGR and Fetal outcome. 


\section{International Journal of Science and Research (IJSR) \\ ISSN (Online): 2319-7064}

Index Copernicus Value (2015): 78.96 | Impact Factor (2015): 6.391

Oligohydramnios is more commonly associated with intra uterine growth restriction. The mechanism behind oligohydramnios is uteroplacental insufficiency and reduced renal perfusion. This is consistent with the findings observed in this study. In Doppler CTG group, $76 \%$ and in CTG alone group $66 \%$ were associated with oligohydramnios.

The mean gestational age in this study is 35 weeks. 35 out of 50 in the Doppler and CTG group were terminated at 34-36 weeks with antenatal steroids administration based on the Doppler abnormality detected.

In CTG alone group, 26 out of 50 were terminated > 36 weeks and 22 were terminated at 34-36 weeks. This show that the Doppler identifies the early hypoxic changes and act as an effective surveillance tool when compared to CTG which identifies at later period. In similar studies conducted by DHARMAVIJAYA ET AL showed the mean gestational age of birth as around 34 weeks. In study by HALEY ET $\mathrm{AL}$, it was 38-39 weeks.

Mode of delivery is very important in the outcome of IUGR fetuses. In this study, $94 \%$ in Doppler CTG group and $72 \%$ in the CTG alone group underwent cesarean section. Cesarean section rate is higher in both the study groups. This is consistent with findings observed in the study conducted by DHARMAVIJAYA ET AL. cesarean is the preferred mode of delivery in the intra uterine growth restricted fetuses with early hypoxic changes to prevent the neonatal morbidity and mortality.

The birth weight observed in this study was that $58 \%$ was below $2 \mathrm{~kg}$ in both the groups.In similar study by CHAKRABORTY ET AL, the mean birth weight was 2.6 $\mathrm{kg}$ and by HALEY ET AL, it was $2.5 \mathrm{~kg}$.

In this study, $82 \%$ of the Doppler and CTG group, and $90 \%$ of the CTG alone group had APGAR at birth $>7$.this is comparable to the mean APGAR in the study conducted by HALEY ET AL was 9. The better surveillance helps inthe early detection of the hypoxic insults thereby deciding timely and optimal intervention to improve the neonatal outcome.

The babies born with low APGAR had longer NICU stay and more associated with neonatal complications. In this study, in CTG alone group, 10 out of 50 had longer NICU stay whereas in Doppler and CTG group, only 2 out of 50 had longer NICU stay > 7 days.Early detection was inversely proportional to the duration of NICU admission. This proves that Doppler ultrasound is more superior than cardiotocography as an effective surveillance tool in IUGR , in predicting adverse neonatal complications and improving the neonatal outcome.

\section{Conclusion}

The Doppler velocimetry and Cardiotocography are primary monitoring tools in the optimal management of Intra uterine growth restricted fetuses. The Cardiotocography identifies only the acute events and Doppler velocimetry identifies the chronic hypoxic insults. Doppler velocimetry identifies the insult earlier than CTG. The Doppler velocimetry when compared with Cardiotocography is more advantageous in the surveillance of intra uterine growth restricted fetuses and in early decision making. The Doppler velocimetry is superior to Cardiotocography in providing information for timely intervention, thereby reducing perinatal morbidity and mortality to a greater extent. Thus, dopplervelocimetry is more effective than Cardiotocography in the surveillance of intra uterine growth restricted fetuses and to predict and reduce the adverse neonatal outcome.

\section{References}

[1] Randomized controlled trial of cardiotocography versus umblical artery Doppler in the management of small for gestational age fetuses, british journal of obstetrics and gynecology, april 1997, vol 104, Haley et al

[2] Journal on antepartum surveillance in intra uterine growth retardation, journal of clinical biomed science 2013, dharmavijaya et al

[3] Evaluation of perinatal outcome by antenatal CTG and umblical artery Doppler in pre eclamptic mothers , indian journal of clinical practice, vol 24, nov 2013, chakraborty et al

[4] Doppler velocimetry and non stress test in severe fetal growth restriction. Clinical expert obstetgynecol, 2001, gramellini et al

[5] Intra utereine growth restriction- screening, diagnosis and management, 295, August 2013,journal of obstetrics and gynecology Canada.

[6] Optimizing the definition of intra uterine growth restriction, the multicenter prospective PORTO study, Unterscheider J, Daly S, Geary MP, et al. Am J ObstetGynecol 2013;208:290.e1-6.

[7] Cardiotocography alone versus cardiotocography plus Doppler evaluation of the fetal middle cerebral and umbilical artery for intrapartum fetal monitoring: a Greek prospective controlled trial.J Matern Fetal Neonatal Med. 2012 Jul;25(7. Kassanos et al

[8] 2 year neurodevelopmental and intermediate perinatal outcomes in infants with very preterm fetal growth restriction (TRUFFLE): a randomised trial, lees et al

[9] The growth restriction intervention trial; long term outcomes in a randomised trial of timing of delivery in fetal growth restriction, walker et al, American journal of obsgynec, jan 2011

[10] Progression of Doppler abnormalities in IUGR, toren et al, Ultrasound ObstetGynecol2008; 32: 160-16

[11] Baschat AA. Fetal responses to placental insufficiency: an update. BJOG 2004; 111: 1031-1041.

[12] Intrauterine growth restriction: new concepts in antenatal surveillance, diagnosis, and management FrancescFigueras, MD, PhD; Jason Gardosi, MD, AJOJ

[13] Kady S, Gardosi J. Perinatal mortality and fetal growth restriction. Best Pract Res ClinObstetGynaecol 2004; 18:397-410

[14] Pattison N, McCowan L. Cardiotocography for antepartum fetal assessment. Cochrane Database Syst Rev 2000;(2):CD001068. Update in: Cochrane Database Syst Rev 2010;(2):CD001068.

[15] Alfirevic Z, Stampalija T, Gyte GM. Fetal and umbilical Doppler ultrasound in high-risk pregnancies. Cochrane Database Syst Rev 2010;(1):CD007529 


\section{Author Profile}

Dr. S. S. Gayathri M.D(OG), DNB(OG) is Assistant Professor of

Obstetrics and Gynecology, Madurai Medical College, Madurai.

Dr. T. H. Usha M.D (OG), DNB(OG) is Assistant Professor of

Obstetrics and Gynecology, Madurai Medical College, Madurai 